\title{
Degradación de Residuos de Pirimetanil en Frutilla (Fragaria x Ananassa Duch.)
}

\author{
Bibiana Diaz ${ }^{(1)}$, Olga N. Saavedra ${ }^{(2)}$, Susana Borkosky ${ }^{(3)}$ y Maria G. Bovi Mitre ${ }^{(2) *}$ \\ (1) Universidad Nacional de Tucumán, Facultad de Agronomía y Zootecnia, \\ Av. Roca 1800, (4000) S. M. de Tucumán-Argentina. \\ (2) Universidad Nacional de Jujuy, Grupo INQA, Facultad de Ingeniería, \\ Gorriti 237, (4600) S.S de Jujuy- Argentina. \\ (3) Universidad Nacional de Tucumán, Facultad de Bioquímica, Química y Farmacia, \\ Ayacucho 471, (4000) S. M. de Tucumán- Argentina.
}

* autora a quien debe ser dirigida la correspondencia

\section{Resumen}

Se investigó la degradación del funguicida Pirimetanil en Fragaria $x$ ananassa Duch. cultivada en Tucumán, Argentina. Se realizaron dos tratamientos con cuatro repeticiones y se usaron las dosis máximas y mínimas recomendadas en la literatura. Los frutos cosechados se extrajeron con solventes orgánicos y en los extractos obtenidos se cuantificó el Pirimetanil empleando cromatografía líquida-líquida de alta resolución. En el tratamiento que recibió la dosis máxima los residuos encontrados a los 11 días fueron $1.72 \mathrm{mg} / \mathrm{kg}$. Esta situación es diferente cuando se aplica la dosis mínima, debido a que en el tercer día se registran valores inferiores al límite máximo de residuos $(1 \mathrm{mg} / \mathrm{kg}$.). El período de carencia para la región del ensayo es diferente al establecido por la legislación argentina, lo que evidencia la necesidad de realizar análisis en cada zona agro-ecológica.

Palabras claves: fragaria, pirimetanil, funguicida, curvas de degradación

\section{Degradation of Pyrimethanil in Strawberries (Fragaria $x$ ananassa Dutch.)}

\begin{abstract}
The degradation of the fungicide Pyrimentanil in a Fragaria $x$ ananasse Duch crop in the Province of Tucumán, Argentina was investigated. Two treatments were done with four repetitions and the maximum and minimum doses recommended in the literature were used. Harvested fruits were removed with organic solvents and Pirimetanil was quantified in the extracts using high resolution liquid-liquid chromatography. In the treatment where the maximum dose was applied, the residues found on the $11^{\text {th }}$ day were $1.72 \mathrm{mg} / \mathrm{kg}$. This situation is different when the minimum dose is applied, since on the third day residue values below the maximum limit were registered $(1 \mathrm{mg} / \mathrm{kg})$. The lack period for the test region is different from that established by argentinean legislation, making evident the need of performing analyses in each agro-ecological area.
\end{abstract}

Keywords: fragaria, pyrimethanil, fungicide, degradation curves 


\section{INTRODUCCIÓN}

La provincia de Tucumán es la principal productora de frutillas del norte argentino. El departamento de Lules es la región productora por excelencia de "frutilla primicia" que se comercializa en los meses de mayo, junio, julio y agosto. Esta provincia garantiza todo el año su presencia en el mercado para consumo fresco, industrialización y exportación de fruta congelada.

La frutilla es un cultivo muy variable en cuanto a su morfología y uno de los más ampliamente adaptados en el mundo. La planta de interés comercial, Fragaria $x$ ananassa Duch. pertenece taxonómicamente a la familia Rosaceae, tribu Potentilla (Gambardella, 1996). La mayoría de las enfermedades que afectan este fruto en Tucumán, están relacionadas con hongos, como es el caso del moho gris (Botritis cinerea) que ocasiona importantes pérdidas. El hongo ataca todos los órganos aéreos, pero pudre principalmente el fruto en la pre y poscosecha, lo que implica la aplicación de funguicidas para su control. El uso continuo de agroquímicos trae aparejado la resistencia de los patógenos a los principios activos. Esta situación obliga a emplear compuestos químicos nuevos que presenten modos de acción diferentes a los ya existentes.

El Instituto para ensayos con plaguicidas en Alemania presentó en el año 2002 un funguicida nuevo, el Pirimetanil y autorizó su uso en frutilla para el control de $B$. cinerea. Numerosos trabajos recientes aportan datos respecto al tratamiento fitosanitario con Pirimetanil. Garau et al. (2002) estudiaron la degradación de este funguicida en cultivos de tomates de invernadero y establecieron que el mecanismo de disipación está influenciado por procesos como la fotodegradación. Milling et al. (1996) determinaron que impide la secreción de las proteínas del patógeno necesarias para las etapas iniciales de la infección, por reducción de los niveles de algunas enzimas hidrolíticas. Concluyeron que su modo de acción es sistémico, translaminar, preventivo y curativo.

Verdisson et al. (2001) midieron la toxicidad de este fitoterápico en plantas acuáticas y los resultados mostraron que el alga Lemna minor fue muy sensible al fungicida. Otros autores han encontrado que además de inhibir el desarrollo de $B$. cinerea en vid, el fungicida tiene un efecto benéfico sobre la fisiología de los hidratos de carbono (Saladin et al. 2003)

Aplicado el plaguicida experimenta cambios debido a las condiciones climatológicas y a la interacción con el órgano vegetal, lo que provoca una disipación del mismo a una velocidad determinada. Las plantas degradan los plaguicidas por reacciones de oxidación, reducción, hidrólisis y combinación con glucosa, aminas y glutatión En muy pocos casos la degradación termina en $\mathrm{CO}_{2}$ y $\mathrm{H}_{2} \mathrm{O}$. En otros casos ocurre la excreción de metabolitos que se acumulan en el vegetal. Estudios con derivados sustituidos con ${ }^{14} \mathrm{C}$ indican que plaguicidas aromáticos o heterocíclicos no se fijan fácilmente al vegetal pero quedan como metabolitos conjugados solubles o como residuos insolubles (Frea, 1976).

La cantidad de plaguicida que queda sobre un vegetal después de una aplicación, depende, entre otros factores, de la dosis y es necesario conocer como varían estos residuos en función del tiempo, para determinar el período de "tolerancia". Así se determina el "plazo de seguridad" que debe transcurrir entre el tratamiento y la recolección (Coscollá, 1993).

No hay homogeneidad entre las diferentes legislaciones acerca de límites máximos de residuos de plaguicidas (LMR), aunque en algunos países se están tomando medidas para tratar de armonizar estas discrepancias (Moran y García, 2002; Alonso Díaz, 2001). En la Comunidad Económica Europea, el LMR para el Pirimetanil varía desde $1 \mathrm{mg} / \mathrm{kg}$ en Francia, a $5 \mathrm{mg} / \mathrm{kg}$ en España y Alemania. Estados Unidos establece el LMR de $3 \mathrm{mg} / \mathrm{kg}$ (U. S. EPA, 2004). En Argentina, la legislación establece un periodo de carencia de 8 días y un LMR de $1 \mathrm{mg} / \mathrm{kg}$ (SENASA, 2005).

El objetivo del presente trabajo es: Obtener experimentalmente las curvas de degradación (Pirimetanil: frutilla) para las dosis máxima y mínima recomendadas, en la zona de Lules (Tucumán).

\section{PROCEDIMIENTO EXPERIMENTAL}

\section{Materiales}

El nombre Pirimetanil corresponde al compuesto químico que según I.U.P.A.C es el N- 
(4,6-dimetilpirimidin-2-il) anilina, de fórmula molecular $\mathrm{C}_{12} \mathrm{H}_{13} \mathrm{~N}_{3}$ y peso molecular 199. Es un sólido cristalino blanco, PF $96,3^{\circ} \mathrm{C}$ y tiene una solubilidad en agua de $0,121 \mathrm{~g} / \mathrm{l}$ a $25^{\circ} \mathrm{C}$.

\section{Metodología}

El ensayo de campo se implantó en el mes de Junio, en una explotación de producción comercial de frutillas (Fragaria $x$ ananassa Duch.) del cultivar Camarosa ubicada en el Km19 de la ruta 301, Departamento Lules de la Provincia de Tucumán (Argentina).

El diseño experimental utilizado fue de bloques completos aleatorizados, con 2 tratamientos y 4 repeticiones, la unidad experimental fue de $10 \mathrm{~m} \times 1 \mathrm{~m}\left(10 \mathrm{~m}^{2}\right)$

\section{Los tratamientos fueron:}

T1: tres aplicaciones previas a la cosecha con un intervalo de 15 días. Las aplicaciones se realizaron el 20/VI, el 5 y el 25/VII. La dosis utilizada fue de $500 \mathrm{~g}$ i.a /ha.

T2: tres aplicaciones previas a la cosecha con un intervalo de 15 días. Las aplicaciones se realizaron el los mismos días que en el T1. La dosis utilizada fue de $1000 \mathrm{~g}$ i.a /ha

Testigo: se dejó un bloque para cosechar frutillas sin aplicaciones del producto, para ajustar el método analítico y determinar la recuperación del método químico.

Se utilizó una mochila de presión constante a una presión de 40 libras (360 mL/min), con boquilla tipo Cone Jet, TXA8001VK. La cosecha se inició el día 25/VII, según la práctica normal.

Se cosecharon al azar frutos de distintos bloques de la parcela, a los $3,6,11,15$ y 28 días desde del inicio de la cosecha. Debido a que los productores, no realizan la cosecha el mismo día de la aplicación de plaguicidas, se consideró como punto inicial de la curva el tercer día desde la última aplicación

Se registraron los datos climáticos: temperatura, precipitaciones, humedad relativa y heliofanía.

Se realizaron los análisis según la técnica analítica propuesta por Wrede (1994): trituración de la muestra; obtención de extractos mediante la maceración de la fruta triturada con acetato de etilo; lavado con solución $0.2 \mathrm{M}$ de $\mathrm{NaOH}$. Se decantó y la capa orgánica concentrada se cromatografió en columna con silica gel eluyendo con nhexano: acetato de etilo 3:7. El eluato se purificó con SPE (extracción en fase sólida) empleando cartuchos C18. Se eluyó con acetonitrilo y se concentró en rotavapor

El extracto concentrado se retomó con $2 \mathrm{ml}$ de acetonitrilo:agua (60:40), se filtró con membrana de Nylon de 0.45 micrones y se analizó con un cromatógrafo líquido Konik 500 con inyector Reodyne y detector ultravioleta a 268 nm, Columna Nucleosil $C_{18}$, Fase móvil acetonitrilo:agua (60:40). Límite de detección: $0,05 \mathrm{mg} / \mathrm{kg}$. Los valores de residuos fueron corregidos según la recuperación del método (90\%). Se realizaron por cuadruplicado y fueron analizados estadísticamente con el programa SPSS.

\section{RESULTADOS Y DISCUSIÓN}

En el tratamiento que recibió 3 aplicaciones del funguicida, con la dosis máxima (T2) los residuos encontrados a los 11 días, fueron $1.72 \mathrm{mg} / \mathrm{kg}$. Se supera el periodo de carencia establecido por la legislación argentina para este funguicida. Esta situación es diferente para el tratamiento 1 ya que cuando se aplica la dosis mínima, al tercer día se registran valores inferiores al LMR (Fig. 1).

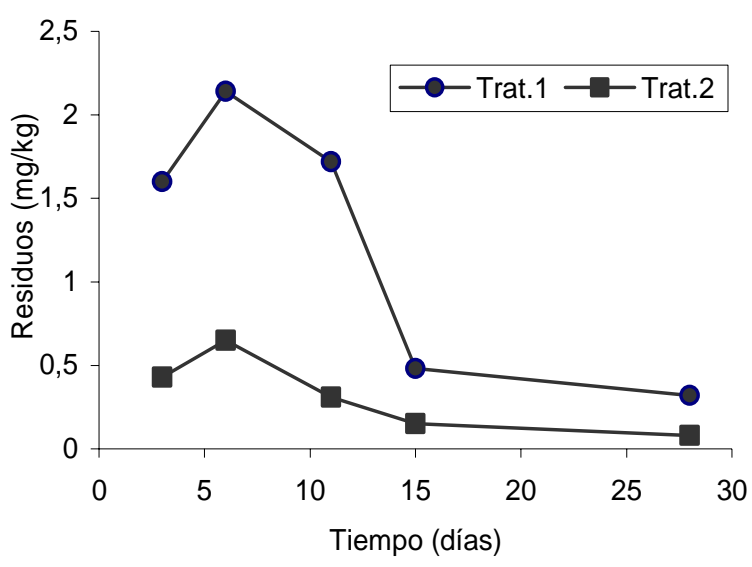

Fig. 1: Curvas de degradación para el par Pirimetanil: frutilla para las dosis máxima y mínima recomendadas.

En coincidencia con lo que mencionan diversos autores, se observa que el depósito inicial es mayor cuando se aumenta la dosis. En este estudio, al duplicarse la misma, los residuos cuadruplicaron su valor. 
Los resultados muestran concentraciones de residuos de $0,32 \mathrm{mg} / \mathrm{Kg}$ y $0,07 \mathrm{mg} / \mathrm{Kg}$ a los 28 días de iniciada la cosecha, para las condiciones del ensayo y las dos dosis.

Los resultados determinados experimentalmente se registran en la Tabla 1 y se grafican en la Figura 1.

Tabla 1- Residuos de Pirimetanil en frutilla tratadas con la dosis máxima y mínima

\begin{tabular}{|c|c|c|c|c|}
\hline \multirow{2}{*}{ Dias } & \multicolumn{2}{|c|}{$\begin{array}{c}\text { T1-Dosis máxima } \\
(1000 \mathrm{~g} / \mathrm{ha})\end{array}$} & \multicolumn{2}{c|}{$\begin{array}{r}\text { T2-Dosis mínima } \\
(500 \mathrm{~g} / \mathrm{ha})\end{array}$} \\
\cline { 2 - 5 } & $\mathrm{mg} / \mathrm{kg}$ & $\begin{array}{c}\mathrm{mg} / \mathrm{kg} \\
\text { promedio }\end{array}$ & $\mathrm{mg} / \mathrm{kg}$ & $\begin{array}{c}\mathrm{mg} / \mathrm{kg} \\
\text { promedio }\end{array}$ \\
\hline \multirow{4}{*}{3} & 1.60 & & 0.47 & \\
& 1.60 & 1.60 & 0.47 & 0.43 \\
& 1.58 & & 0.47 & \\
& 1.62 & & 0.32 & \\
\hline \multirow{5}{*}{6} & 2.14 & & 0.63 & \\
& 2.16 & 2.14 & 0.67 & 0.65 \\
& 2.10 & & 0.65 & \\
& 2.14 & & 0.65 & \\
\hline \multirow{5}{*}{11} & 1.72 & & 0.33 & \\
& 1.72 & 1.72 & 0.32 & 0.31 \\
& 1.70 & & 0.31 & \\
& 1.74 & & 0.28 & \\
\hline \multirow{4}{*}{15} & 0.48 & & 0.17 & \multirow{2}{*}{0.14} \\
& 0.46 & 0.48 & 0.15 & \\
& 0.50 & & 0.13 & \\
& 0.48 & & 0.13 & \\
\hline \multirow{3}{*}{28} & 0.32 & & 0.08 & \multirow{2}{*}{0.07} \\
& 0.32 & 0.32 & 0.07 & 0.07 \\
& 0.30 & & 0.07 & \\
& 0.33 & & 0.06 & \\
\hline
\end{tabular}

\section{CONCLUSIONES}

La concentración de residuos presente en los frutos de frutilla después de la aplicación del funguicida y su declinación en el tiempo, depende de la dosis. El periodo de carencia para la región del ensayo es diferente al establecido por la legislación argentina, lo que evidencia la necesidad de realizar los análisis correspondientes en cada zona agroecológica.

\section{REFERENCIAS}

Coscollá, R. "Residuos de Plaguicidas en Alimentos Vegetales", $1^{\mathrm{a}}$ ed. Editor MundiPrensa Libros, Madrid, España (1993).
Frea, D.S., Pesticide conjugate-glicosides in: Bound and conjugate pesticide residues, Kauffman D.D.; Still G.G.; Paulson G.D. and Baudals, S.K. Eds, ACS Symposium Series 29, American Chemical Society, Washington D.C. 35-53 (1976)

Gambardella, M. El cultivo de la frutilla. Publicaciones Misceláneas Agrícolas, Escuela Agronomía, Univ. de Chile, 44:1-6 (1996)

Garau, V.L., A. Angioni , A.A. Del Real, M. Russo y P. Cabras, Disappearance of azoxystrobin, pyrimethanil, cyprodinil, and fludioxonil on tomatoes in a greenhouse, $\mathrm{J}$. Agric. Food Chem., 50 (7):1929-1932. Cagliari, Italy (2002).

Milling, R.J.; A. Daniels y J.B. Pillmoor, The mode of action of the anilinopyrimidines: a new fungicide target, Pesticide Science 47:201-209 (1996)

Moran, A. H. y García, A. E. La armonización del Proceso de Registro y Revisión de Plaguicidas en la Unión Europea y sus implicancias para el mercado de Plaguicidas. XI Jornadas Fitosanitarias. Río Cuarto. Argentina.220 p. (2002)

Saladin, G., C. Magne y C. Clement, Effects of fludioxonil and pyrimethanil, two fungicides used against Botrytis cinerea, on carbohydrate physiology in Vitis vinifera L., Pest. Manag. Sci. 59 (10):1083-1092 (2003)

U. S. Enviromental Protection Agency. Federal Register Enviromental Documents. Pyrimethanil Pesticides Tolerances [fecha de consulta 20 setiembre 2006] (http://www.epa.gov/fedrgstr/EPA-PEST/2004/ August/Day-26/p19525:htm)

Verdisson, S., M. Couderchet y G. Vernet, Effects of procymidone, fludioxonil and pyrimethanil on two non-target aquatic plants, Chemosphere 44 (3):467-474 (2001)

Wrede, A., Pyrimethanil, analytical method for determination of residues in vegetales and fruits by HPLC. Report R/V 2/94 (unveröffentlichter Beritcht, AgrEvo, Berlin) (1994) 